\title{
Filigrane
}

Écoutes psychothérapiques

\section{Les oripeaux des ados. La nouvelle condition subjective des jeunes, de Louise L. Tassé \\ Louise L. Tassé, Les oripeaux des ados. La nouvelle condition \\ subjective des jeunes, Paris, L'Harmattan, 2011}

\section{Monique Lévesque}

Volume 21, numéro 1, printemps 2012

URI : https://id.erudit.org/iderudit/1012884ar

DOI : https://doi.org/10.7202/1012884ar

Aller au sommaire du numéro

Éditeur(s)

Revue Santé mentale au Québec

ISSN

1192-1412 (imprimé)

1911-4656 (numérique)

Découvrir la revue

Citer ce compte rendu

Lévesque, M. (2012). Compte rendu de [Les oripeaux des ados. La nouvelle condition subjective des jeunes, de Louise L. Tassé / Louise L. Tassé, Les oripeaux des ados. La nouvelle condition subjective des jeunes, Paris, L’Harmattan, 2011].

Filigrane, 21(1), 119-121. https://doi.org/10.7202/1012884ar 


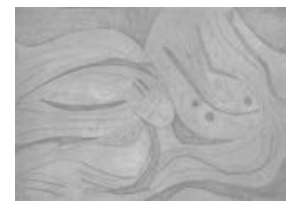

\section{Les oripeaux des ados. La nouvelle condition subjective des jeunes, de Louise L. Tassé ${ }^{1}$}

\section{Monique Lévesque}

- oute œuvre majeure porte en elle le pouvoir de faire résonner chez le lecteur un espace déjà en attente d'interlocution.

Louise L. Tassé par cet ouvrage nous fait le plus grand don que l'on puisse recevoir, à savoir celui d'un espace pour «La Pensée » : ainsi elle dégage de la gangue de la mollesse, du je-m'en-foutisme et du laisser-faire ambiant, un questionnement profondément empreint de gravité, d'une étonnante acuité et surtout d'une indubitable dimension éthique. Ce qui a supposé un immense travail d'élaboration et de recensement des théories promouvant l'accès à une véritable subjectivation pour tout sujet humain, à savoir la théorie psychanalytique, l'anthropologie et l'épistémologie, le tout coiffé d'une disponibilité à l'altérité de la parole de l'autre.

Au sein de l'extrême densité de son texte, j'ai souhaité prélever trois thèmes qui me sont apparus extrêmement cruciaux à savoir ;

- le statut de la langue et une praxis de la parole;

- le déboulonnement du primat de la statistique;

- la subversion de l'idéologie du scientisme assaisonné à la sauce néolibérale, subversion opérée à la lumière de questionnements épistémiques incontournables, pour qui veut bien s'autoriser à penser aujourd'hui.

Donc trois axes: disponibilité à la langue, disponibilité à la sensorialité qualitative, et éthique de l'expérience, le tout majestueusement articulé à une sûreté épistémique sans faille, ce qui a pour effet une désaliénation de l'idéologie dominante.

La langue, en tant qu'énonciation subjective, est le fondement de la vie psychique pour tout être humain et constitue l'élément structural qui permet à notre statut de sujet de s'instaurer. La langue constitue de la corporéité en voie d'émergence, de la corporéité sous sa forme native et vagissante, tout 
autant qu'elle peut aussi être transformée en processus de néantisation d'un sujet.

Louise L. Tassé a rencontré des sujets en déshérence, des êtres parlants au sein de leur détresse affective et de leur carence d'interlocution, et elle a consenti à ouvrir le dialogue avec chacun d'entre eux. Pour être très brève, je dirais que parler et être écouté se révèle assez souvent être un enjeu de vie et de mort. C'était le point 1: reconnaître la matérialité psychique de la parole et de la langue.

Passons au point 2: la mise en échec de la statistique comme outil de recension et de connaissance, supposée explicative face à la prégnance du «malêtre» touchant et mettant en péril les conditions de subjectivation d'adolescents laissés à eux- mêmes. La statistique, n'est-ce pas là un magnifique anesthésiant pour la pensée? Répertorier des items, les assembler en colonnes de chiffres, relever des pourcentages selon les échelles d'âge et de statuts socioéconomiques, n'implique d'aucune façon, loin de là, une prise en compte de la nécessité structurale d'une relation intersubjective. Cette approche n'implique pas non plus la possibilité d'un lien, notamment la présence du TIERS comme élément de médiation symbolique, lequel a le pouvoir de présider à une véritable rencontre avec autrui. Pourrait-on établir le triste constat que le plus souvent, la performance épidémiologique a réussi à évacuer toute potentialité de rencontre avec l'autre?

Louise L. Tassé, anthropologue et chercheure, nous démontre par la rigueur de son texte que l'analyse critique des conditions subjectives et sociétales éclaire, indique et clame haut et fort, à qui veut bien l'entendre, la carence interlocutoire, le déficit d'un espace de symbolicité, le désarrimage intergénérationnel et la quête du sacré de jeunes adolescents. Toutes ces conditions se révèlent éminemment présentes et prégnantes chez certains jeunes en difficulté de filiation. Ce savoir ne peut découler que d'une recherche qui se déploie dans un autre lieu que celui du quantitatif positiviste et du recensement réducteur à visée d'obturation des véritables enjeux.

Point 3 : peut-on échapper à cette " perversion ordinaire ${ }^{2}$ ", mise à nu de la logique du Même et/ou de la répétition mortifère? Peut-on échapper au diktat de la décérébration bureaucratique institutionnalisée ? À la lecture du texte de Louise L. Tassé, il apparaît que oui. Un être vraiment vivant est nécessairement mû et mobilisé par une incessante et inéluctable nécessité de PENSER, tout autant que par une nécessité d'interrogation, afin de suivre à la trace toute étincelle d'intelligence, toute proposition qui ouvre une piste. La fréquentation de quelques chamans théoriciens (Marcel Gauchet, Alain 
Didier-Weil, Dany-Robert Dufour, Suzanne Ginestet-Delbreil, Jacques Lacan, Jean-Pierre Lebrun, Claude Lévi-Strauss...) puis la réélaboration de leur apport, autorise la fulgurance du saisissement d'une problématique et l'instauration d'une tonalité d'appréhension juste et éthique. Tout cela, Louise L. Tassé nous l'offre, par la somme d'un patient et long travail de réflexion, de décodage, de métabolisation théorique et de véritable écoute, ainsi que par le biais de l'oreille d'une musicienne.

Les oripeaux des ados fait s'écrouler les statistiques fantoches, les fauxsemblants de scientificité, lesquels pullulent au cour des sciences humaines nord-américaines tout autant qu'ailleurs. Les idoles biologisantes se désintègrent de même que leurs avatars, à savoir la tromperie, le mensonge et l'abêtissement à tout crin. Je vous invite donc à goûter un élixir d'intelligence, de rigueur et de foi en l'acte de parole, tout autant qu'en celui de l'écoute de l'Autre!

Monique Lévesque moniquelevesque@gmail.com

\section{Note}

1. Louise L. Tassé, Les oripeaux des ados. La nouvelle condition subjective des jeunes, Paris, L'Harmattan, 2011.

2. La perversion ordinaire. Vivre ensemble sans autrui est le titre d'un ouvrage récent de JeanPierre Lebrun paru à Paris chez Denoël en 2007. 